\title{
Associação entre perfil socioeconômico e estado nutricional em adultos com excesso de peso
}

Association between socioeconomic profile and nutritional state in adults with overweight Asociación entre perfil socioeconómico y estado nutricional en adultos con sobrepeso

Rodolfo Felipe Lima Coelho ORCID: https://orcid.org/0000-0002-7547-461X Universidade Estadual do Paraná, Brasil

E-mail: rodolfofelipe@ hotmail.com

Heloá Costa Borim Christinelli

ORCID: https://orcid.org/0000-0003-0772-4194

Universidade Estadual de Maringá, Brasil

E-mail: heloa.borim@hotmail.com

Greice Westphal

ORCID: https://orcid.org/0000-0001-9107-0108 Universidade Estadual de Maringá, Brasil

E-mail: greicewes@gmail.com

Maria Luiza Costa Borim

ORCID: https://orcid.org/0000-0002-9523-4218 Universidade Estadual de Maringá, Brasil

E-mail: luborim10@hotmail.com

Raquel Tomiazzi Utrila

ORCID: https://orcid.org/0000-0001-9454-2713 Universidade Estadual de Maringá, Brasil

E-mail: raquelutrila@gmail.com

Igor Alisson Spagnol Pereira

ORCID: https://orcid.org/0000-0001-7340-3909

Universidade Estadual de Maringá, Brasil

E-mail: igorspagnol2@hotmail.com

Fernando Malentaqui Martins

ORCID: https://orcid.org/0000-0003-1623-2183

Universidade Estadual de Maringá, Brasil

E-mail: nandoesporte1@ gmail.com

Mario Moreira Castilho

ORCID: https://orcid.org/0000-0002-4855-8236

Universidade Estadual de Maringá, Brasil

E-mail: mmcastilho_1905@hotmail.com

Geison Schmidt Soares

ORCID: https://orcid.org/0000-0001-5150-6931

Universidade Estadual de Maringá, Brasil

E-mail: geisonssoares@gmail.com

Michele Fernandes do Amaral

ORCID: https://orcid.org/0000-0002-4812-9773

Universidade Estadual de Maringá, Brasil

E-mail: micheleamaralpsico@gmail.com

Maria Antonia Ramos Costa

ORCID: https://orcid.org/0000-0001-6906-5396

Universidade Estadual do Paraná, Brasil

E-mail: maria.costa@unespar.edu.br

Nelson Nardo Junior

ORCID: https://orcid.org/0000-0002-6862-7868

Universidade Estadual de Maringá, Brasil

E-mail: nnjunior@uem.br

Carlos Alexandre Molena Fernandes

ORCID: https://orcid.org/0000-0002-4019-8379

Universidade Estadual de Maringá, Brasil

E-mail: carlosmolena126@gmail.com 


\title{
Resumo
}

Introdução: No Brasil, a prevalência do excesso de peso e obesidade é maior entre os indivíduos com menor escolaridade, $24,5 \%$ de obesidade entre os que têm de 0 a 8 anos de escolaridade e de $15,8 \%$ no grupo com 12 anos ou mais de escolaridade. Objetivo: o objetivo deste estudo foi analisar a associação entre o perfil socioeconômico e o estado nutricional de adultos com excesso de peso no município de Paranavaí - PR. Método: Trata-se de um estudo descritivo transversal destinado a identificar os fatores associados ao estado nutricional: nível de renda e escolaridade; em adultos, de ambos os sexos, com idade entre 18 e 50 anos, com excesso de peso. Resultados: Dentre os 147 participantes a média de idade apresentada foi de 34,52 anos 87 eram casados, 54 solteiros, 5 divorciados e 1 viúvo, no tocante ao sexo 119 eram mulheres, a maioria apresentou 8 ou mais anos de estudo, prevaleceram participantes com a Obesidade Grau I com 32,65\%. Conclusão: O enfermeiro como gestor de uma unidade básica de saúde tem papel fundamental no desenvolvimento de atividades de educação em saúde visando a mudança do estilo de vida para a prevenção e tratamento da obesidade.

Palavras-chave: Nível socioeconômico; Saúde; Estado Alimentar; Alimentação Saudável.

\begin{abstract}
Introduction: In Brazil, the prevalence of overweight and obesity is higher among individuals with less education, $24.5 \%$ of obesity among those with 0 to 8 years of schooling and $15.8 \%$ in the group with 12 years or more education. Objective: the objective of this study was to analyze the association between the socioeconomic profile and the nutritional status of overweight adults in the municipality of Paranavaí - PR. Method: This is a descriptive crosssectional study designed to identify the factors associated with nutritional status: level of income and education; in adults, of both sexes, aged between 18 and 50 years, with excess weight. Results: Among the 147 participants, the average age presented was 34.52 years old. 87 were married, 54 single, 5 divorced and 1 widower, with regard to sex 119 were women, most had 8 or more years of study, participants prevailed. with Grade I Obesity with $32.65 \%$. Conclusion: The nurse as a manager of a basic health unit has a fundamental role in the development of health education activities aimed at changing the lifestyle for the prevention and treatment of obesity.
\end{abstract}

Keywords: Socioeconomic level; Health; Food status; Food Health.

\begin{abstract}
Resumen
Introducción: En Brasil, la prevalencia de sobrepeso y obesidad es mayor entre los individuos con menos educación, $24,5 \%$ de obesidad entre los de 0 a 8 años de escolaridad y 15,8\% en el grupo de 12 años. o más educación. Objetivo: el objetivo de este estudio fue analizar la asociación entre el perfil socioeconómico y el estado nutricional de adultos con sobrepeso en el municipio de Paranavaí - PR. Método: Se trata de un estudio descriptivo transversal diseñado para identificar los factores asociados al estado nutricional: nivel de ingresos y educación; en adultos, de ambos sexos, de entre 18 y 50 años, con sobrepeso. Resultados: Entre los 147 participantes, la edad promedio presentada fue de 34,52 años, 87 eran casados, 54 solteros, 5 divorciados y 1 viudo, con respecto al sexo 119 eran mujeres, la mayoría tenía 8 o más años de estudio, prevalecieron los participantes. con Obesidad Grado I con 32,65\%. Conclusión: La enfermera como gerente de una unidad básica de salud tiene un papel fundamental en el desarrollo de las actividades de educación en salud orientadas a cambiar el estilo de vida para la prevención y el tratamiento de la obesidad.
\end{abstract}

Palabras clave: Nivel socioeconómico; Salud; Situación Alimentaria; Comida Saludable.

\section{Introdução}

Uma interação entre fatores biológicos, genéticos, ambientais, sociais e psicológicos levam a um balanço energético positivo e, consequentemente, ao ganho de peso (Mussi \& Petróski, 2019). Dentre estes fatores, pesquisas têm destacado a associação entre obesidade e aspectos socioeconômicos como renda e escolaridade (Ferraz et al., 2019).

Dados da última pesquisa Vigilância de Fatores de Risco e Proteção para Doenças Crônicas por Inquérito Telefônico (VIGITEL) mostram que, no Brasil, a prevalência do excesso de peso e obesidade é maior entre os indivíduos com menor escolaridade. Assim, a prevalência de $24,5 \%$ de obesidade entre os que têm de 0 a 8 anos de escolaridade enquanto isso a prevalência foi de 15,8\% no grupo com 12 anos ou mais de escolaridade (Brasil, 2019).

Tem-se que indivíduos em ambientes com poucos recursos e de maior vulnerabilidade e menos favorecidos apresentam maior chance de exposição a produtos e práticas não saudáveis e assim maior chance de desenvolver fatores de risco metabólicos para o desenvolvimento de Doenças Cardiovasculares (DCV) (WHO, 2018).

Sendo as doenças crônicas não transmissíveis (DCNT) muito prevalentes, multifatoriais e com coexistência de determinantes biológicos e socioculturais, a sua abordagem, para ser efetiva, necessariamente envolve as diversas categorias profissionais das equipes de saúde e exige o protagonismo dos indivíduos, suas famílias e comunidade (Brasil, 2014). 
O enfermeiro como gestor da atenção primaria à saúde (APS), tem o papel de realizar ações no âmbito da promoção da saúde, prevenção de agravos e recuperação da saúde; sendo capaz de compreender o contexto indispensável à superação dos problemas de saúde locais, inclusive a prevenção e o controle da obesidade (Nicolau Ian Rigon; Santos, 2017). Diante do exposto, o objetivo deste estudo foi analisar a associação entre o perfil socioeconômico e o estado nutricional de adultos com excesso de peso no município de Paranavaí - PR.

\section{Metodologia}

Trata-se de um estudo descritivo transversal destinado a identificar os fatores associados ao estado nutricional: nível de renda e escolaridade; em adultos, de ambos os sexos, com idade entre 18 e 50 anos, com excesso de peso, de acordo com os critérios da WHO (WHO, 2017).

O estudo foi divulgado por meio das redes sociais, mídias impressas e faladas, instituições de ensino superior, e unidades básicas de saúde (UBS) de um município da região noroeste. Na divulgação foram informados os telefones, e-mail, site e endereço do Centro de Pesquisa da Universidade responsável pela pesquisa para que os interessados entrassem em contato para receber orientações da equipe sobre o processo de triagem.

Os critérios de inclusão adotados neste estudo foram idade: maior ou igual a 18 anos e menor ou igual a 50 anos; estar com sobrepeso ou obesidade, a partir de pontos de corte do Índice de Massa Corporal (IMC) $\geq 25$ (WHO, 2017) e ser residente no município do estudo.

A coleta de dados ocorreu entre os meses de novembro e dezembro de 2019 e foram avaliados 147 indivíduos para a seleção de amostra, e todos os interessados foram submetidos a avaliação que incluiu medidas antropométricas de peso e estatura para determinar quais atendiam ao perfil elegível para o estudo. Posteriormente a essa avaliação antropométrica, os indivíduos foram classificados conforme o resultado do cálculo do IMC, e os que foram classificados com sobrepeso ou obesidade foram convidados a participar da pesquisa.

Os participantes foram convidados a responder um questionário com vistas a classifica-los quanto ao nível socioeconômico. Para tanto, foi aplicado o questionário de critério de classificação econômica Brasil (ABEP, 2016). Os dados coletados foram organizados em planilha Excel e analisados, por meio do Programa Statistical Package for the Social Sciences (SPSS) versão 20.0 (Andy Field, 2009).

Esta pesquisa faz parte do projeto intitulado: "Eficácia de um programa multiprofissional na avaliação de fatores de risco cardiometabólico e tratamento da obesidade abdominal em dois municípios do noroeste do Paraná", liderado pela Universidade Estadual de Maringá em parceria com a Universidade Estadual do Paraná - Campus Paranavaí, aprovado pelo comitê de ética em pesquisa da Universidade Estadual de Maringá, sob protocolo número 2.655.268.

\section{Resultados}

Dentre os 147 participantes a média de idade apresentada foi de 34,52 anos (mín. 19, máx. 49), em relação ao estado civil 87 eram casados, 54 solteiros, 5 divorciados e 1 viúvo, no tocante ao sexo 119 eram mulheres. Quanto à raça 61 se declararam brancos, 65 pardos, 19 pretos e 2 amarelos.

Em relação ao perfil socioeconômico por IMC, a classe econômica mais prevalente foi "C1". A Tabela 1 apresenta a classificação socioeconômica em relação à classificação do IMC. 
Tabela 1. Classificação socioeconômica de acordo com o IMC dos participantes, Paranavaí, Paraná, Brasil, 2020.

\begin{tabular}{|c|c|c|c|c|c|c|c|c|c|c|c|c|}
\hline \multirow{2}{*}{$\begin{array}{l}\begin{array}{l}\text { Classificação } \\
\text { socioeconômica }\end{array} \\
\text { IMC }\end{array}$} & \multicolumn{2}{|c|}{$\mathbf{A}$} & \multicolumn{2}{|c|}{ B1 } & \multicolumn{2}{|c|}{ B2 } & \multicolumn{2}{|c|}{ C1 } & \multicolumn{2}{|c|}{$\mathrm{C2}$} & \multicolumn{2}{|c|}{ D-E } \\
\hline & $\mathbf{N}$ & $\%$ & $\mathbf{N}$ & $\%$ & $\mathbf{n}$ & $\%$ & $\mathbf{n}$ & $\%$ & $\mathbf{n}$ & $\%$ & $\mathbf{N}$ & $\%$ \\
\hline 25,0 a 29,9 & 2 & 1,4 & 3 & 2,0 & 7 & 4,8 & 2 & 1,4 & 8 & 5,4 & 1 & 0,7 \\
\hline 30,0 a 34,9 & 1 & 0,7 & 5 & 3,4 & 13 & 8,8 & 2 & 1,4 & 9 & 6,1 & 2 & 1,4 \\
\hline 35,0 a 39,9 & 1 & 0,7 & 2 & 1,4 & 12 & 8,2 & 2 & 1,4 & 9 & 6,1 & 2 & 1,4 \\
\hline$\geq 40,0$ & 0 & 0 & 3 & 2,0 & 6 & 4,1 & 7 & 4,8 & 4 & 2,7 & 2 & 1,4 \\
\hline Total & 4 & 2,7 & 13 & 8,8 & 38 & 25,9 & 55 & 37,4 & 30 & 20,4 & 7 & 4,8 \\
\hline
\end{tabular}

Fonte: Autores.

Em relação à escolaridade dos participantes, a maioria apresentou 8 ou mais anos de estudo. A Tabela 2 apresenta a relação da classificação do IMC com a escolaridade dos participantes.

Tabela 2. Características de escolaridade de acordo com o IMC dos participantes, Paranavaí, Paraná, Brasil, 2020.

\begin{tabular}{|c|c|c|c|c|c|c|}
\hline \multirow{2}{*}{$\begin{array}{c}\text { Escolaridade } \\
\text { IMC }\end{array}$} & \multicolumn{2}{|c|}{$>8$ anos de estudo } & \multicolumn{2}{|c|}{$\leq 8$ anos de estudo } & \multicolumn{2}{|c|}{ Total } \\
\hline & $\mathbf{n}$ & $\%$ & $\mathbf{N}$ & $\%$ & $\mathbf{n}$ & $\%$ \\
\hline 25,0 a 29,9 & 6 & 4,1 & 32 & 21,8 & 38 & 25,9 \\
\hline 30,0 a 34.9 & 10 & 6,8 & 38 & 25,9 & 48 & 32,6 \\
\hline 35,0 a 39,9 & 14 & 9,5 & 25 & 17,0 & 39 & 26,5 \\
\hline$\geq 40,0$ & 8 & 5,4 & 14 & 9,5 & 22 & 15,0 \\
\hline Total & 38 & 25,9 & 109 & 74,1 & 147 & 100 \\
\hline
\end{tabular}

Fonte: Autores.

Segundo a classificação do IMC, prevaleceram participantes com a Obesidade Grau I com 32,65\%. A Tabela 3 apresenta a estatística descritiva do IMC dos participantes.

Tabela 3. Prevalência de participantes por classificação do IMC, Paranavaí, Paraná, Brasil, 2020.

\begin{tabular}{lcc}
\hline Classificação & N & \% \\
\hline Sobrepeso & 38 & 25,85 \\
Obesidade_Grau I & 48 & 32,65 \\
Obesidade_Grau II & 39 & 26,53 \\
Obesidade_Grau III & 22 & 14,97 \\
\hline Total & 147 & 100 \\
\hline
\end{tabular}

Fonte: Autores.

Na tabela 4, estão apresentados os resultados da correlação de Spearman das variáveis do índice de massa corporal entre a escolaridade $(r=0.758$ e $\mathrm{p}=0.000)$ e o nível socioeconômico $(r=0.958$ e $\mathrm{p}=0.000)$. Foram encontradas correlações significativas entre as variáveis, apresentando uma forte correlação entre o IMC e as variáveis de Escolaridade e o Nível Socioeconômico, apresentando valor de p significativo.

Tabela 4. Correlação (correlação de Spearman-coeficiente r) entre o índice de massa corporal e a escolaridade e o nível socioeconômico dos participantes, Paranavaí, Paraná, Brasil, 2020.

\begin{tabular}{ccc}
\hline Variáveis & Escolaridade & Nível Socieconômico \\
\hline Índice de Massa Corporal & $\mathrm{r}=0.758^{*}$ & $\mathrm{r}=0.958^{*}$ \\
& $\mathrm{p}=0.000^{*}$ & $\mathrm{p}=0.000^{*}$ \\
\hline
\end{tabular}

Fonte: Autores. 


\section{Discussão}

A classificação social foi baseada na pontuação do questionário socioeconômico, sendo que os participantes foram classificados nos níveis de $\mathrm{A}$ à $\mathrm{E}$, sendo a classe $\mathrm{A}$ a de maior poder aquisitivo e a classe $\mathrm{E}$ a de mais baixo poder aquisitivo (ABEP, 2015; ABEP, 2016). No presente estudo a classificação social com a maior prevalência foi a C1 (37,4\%). No Brasil, 22,2\% da população está classificada como C1 possuindo uma renda média domiciliar de R \$2705,00 segundo a Associação Brasileira de Empresas de Pesquisa (ABEP) em 2016 ( ABEP, 2015; ABEP, 2016). Por sua vez, houve forte correlação entre a escolaridade (maior de oito anos de estudo) com o IMC indicativo de obesidade, diferentemente de outros estudos que apresentaram correlação inversa, ou seja, quanto maior o IMC, menor o nível de escolaridade dos indivíduos (Brasil, 2019; dos Santos Rodrigues; da Silveira, 2015).

Importante ressaltar que a população brasileira gasta, em média, 17,5\% da renda familiar com alimentação, sendo que o consumo de alimentos com baixo teor nutricional e alto teor energético teve um aumento de 56\% de 2003 para 2018 devido ao poder de compra do consumidor (ABEP, 2016; FAO, 2019). Sendo que o aumento do consumo de alimentos pouco saudáveis, aliado aos hábitos sedentários da população, são responsáveis pelo aumento da prevalência do excesso de peso e de doenças crônicas não transmissíveis na população (Martins \& Faria, 2018).

Estudos indicam que a frequência da prática de atividades físicas não está relacionada com a classe social, porém existe uma associação no tipo de atividade física praticada, sendo que pessoas com alto poder aquisitivo frequentam academias e recebem orientações de profissionais de educação física qualificados, enquanto que as classes sociais baixas tem como rotina a prática de atividades físicas por meio de afazeres domésticos e a prática de esportes populares, como por exemplo, futebol (Ferraz et al., 2019; Rodrigues et al., 2017).

Além de ser determinante ao tipo de prática esportiva, o nível de escolaridade e a posição socioeconômica da população estão relacionados também ao acesso aos demais tipos de bens e serviços, principalmente relacionados à alimentação. Ou seja, grupos com menor escolaridade e menor renda tem menor acesso à alimentos saudáveis (Alves \& Faerstein, 2015).

Referente ao preço dos alimentos no Brasil, alguns estudos indicam que produtos naturais e minerais são mais baratos do que os produtos ultra processados, exemplo disto é que um quilo de batata inglesa tem o mesmo preço de um pacote de $200 \mathrm{~g}$ de chips de batata, porém a população prefere a praticidade do que a qualidade (Claro et al., 2016). No entanto, a escolha dos alimentos ultra processados tem como aliado o marketing, visto que o tamanho, a cor, variedades, onde o produto está localizado e até mesmo a imagem passada para o consumidor de se tratar de um alimento saudável influencia no consumo, por exemplo os refrigerantes ou sucos de pacotinho que em suas embalagens contém informações de que são feitos com frutas quando na verdade contém xaropes, aromatizantes e corantes (Souza, 2018).

A globalização é um processo de integração que facilita trocas econômicas, culturais, sociais e políticas. Sendo assim ocasionou mudanças na cultura alimentar mundial por consequência da preocupação com a saúde, falta de tempo levando a crescentes transformações nos hábitos alimentares da população (Resende et al., 2017). Consequentemente houve uma inversão no cenário mundial, a desnutrição que representava um grande desafio de saúde pública, atingiu 821 milhões de pessoas em 2017, enquanto que o sobrepeso e a obesidade atingiram 836 milhões de pessoas em 2016. No Brasil a desnutrição atinge $2 \%$ da população adulta, já a obesidade afeta $20 \%$ da população (FAO, 2019).

\section{Conclusão}

O enfermeiro como gestor de uma unidade básica de saúde tem papel fundamental no desenvolvimento de atividades de educação em saúde visando a mudança do estilo de vida para a prevenção e tratamento da obesidade. Por meio da comunicação 
simples e clara, deve orientar a adoção de uma alimentação saudável, prática de atividade física e acompanhamento nos serviços de saúde para prevenção e tratamento de agravos.

\section{Referências}

ABEP - Associação Brasileira de Empresa de Pesquisas. (2015). Alterações na aplicação do Critério Brasil, válidas a partir de 01/01/2015. ABEP - Associação Brasileira de Empresa de Pesquisas - www.abep.org, 1-5. https://doi.org/10.12820/rbafs.v.6n2p5-18

ABEP - Associação Brasileira de Empresa de Pesquisas. (2016). ASSOCIAÇÃO BRASILEIRA DE EMPRESAS DE PESQUISAS. Critério Brasil 2015 e Alterações na aplicação do Critério Brasil 2016. Associação Brasileira de Empresas de Pesquisa. Critério de classificação econômica Brasil, 1-6. http://www.abep.org/criterio-brasil

Alves, R. F. S., \& Faerstein, E. (2015). Desigualdade educacional na ocorrência de obesidade abdominal: Estudo Pró- Saúde. Revista de Saúde Pública, 49, 18. https://doi.org/10.1590/S0034-8910.2015049005786

Andy Field. (2009). Discovering Statistics using SPSS Statistics. SAGE Publications, 66, 822. http://www.amazon.com/Discovering-Statistics-using-IBMSPSS/dp/1446249182

Brasil. Instituto Brasileiro de Geografia e Estatistica (IBGE). (2019). POF 2017-2018: Famílias com até R\$ 1,9 mil destinam 61,2\% de seus gastos à alimentação e habitação. IBGE. https://agenciadenoticias.ibge.gov.br/agencia-sala-de-imprensa/2013-agencia-de-noticias/releases/25598-pof-2017-2018familias-com-ate-r-1-9-mil-destinam-61-2-de-seus-gastos-a-alimentacao-e-habitacao

Brasil, M. da S. (2014). CADERNOS de ATENÇÃO BÁSICA: Estratégias para o cuidado da pessoa com doenã crônica. In Ministério da Saúde, Secretaria de Atenção à Saúde, Departamento de Atenção Básica (Número 35).

Brasil, M. da S. (2019). Vigitel Brasil 2018: Vigilância de fatores de risco e proteção para doenças crônicas por inquerito telefônico. In G. Estatística e Informação em Saúde. http://bvsms.saude.gov.br/bvs/publicacoes/vigitel_brasil_2011_fatores_risco_doencas_cronicas.pdf

Claro, R. M., Maia, E. G., Costa, B. V. de L., \& Diniz, D. P. (2016). Preço dos alimentos no Brasil: prefira preparações culinárias a alimentos ultraprocessados. Cadernos de Saúde Pública, 32(8). https://doi.org/10.1590/0102-311x00104715

Dos Santos Rodrigues, A. P., \& Da Silveira, E. A. (2015). Correlação e associação de renda e escolaridade com condições de saúde e nutrição em obesos graves. Ciencia e Saude Coletiva, 20(1), 165-174. https://doi.org/10.1590/1413-81232014201.18982013

FAO, F. and A. O. of the U. N. (2019). Dia Mundial da Alimentação faz alerta para o crescimento da obesidade e destaca a alimentação saudável. FAO. http://www.fao.org/brasil/noticias/detail-events/pt/c/1238704/

Ferraz, I. A. R., Pereira, I. G., Monteiro, M. L., Silva, M. de L., Ladeia, A. M., \& Guimaraes, A. (2019). Comparison of the energy and metabolic nutritional profile of women with central obesity of socioeconomic classes a/b vs c/d/e. Nutricion Hospitalaria, 36(4), 819-825. https://doi.org/10.20960/nh.2246

Martins, P. de F. A., \& Faria, L. R. C. (2018). Alimentos ultraprocessados: uma questão de saúde pública. Comunicação em Ciências da Saúde, 29(1), 14-17. http://bvsms.saude.gov.br/bvs/periodicos/ccs_artigos/v29_supl_alimentos_ultraprocessados.pdf\%0Ahttp://www.scielo.br/scielo.php?script=sci_arttext\&pid=S 0034-89102015000100227\&lng=en\&tlng=em

Mussi, R., \& Petróski, E. (2019). Indicadores de obesidade: capacidade preditiva para síndrome metabólica em adultos quilombolas. Ciência \& Saúde Coletiva, 24(7), 2471-2480. https://doi.org/10.1590/1413-81232018247.19032017

Nicolau Ian Rigon; Santo, F. H. do E. C. C. L. de P. (2017). Tratamento multiprofissional para adultos obesos grau III [Tratamiento multidisciplinario para los adultos obesos grado III]. Rev Cubana Enfermer, 33(2), 386-403. http://revenfermeria.sld.cu/index.php/enf/article/view/981/268

World Healh Organization (2018). The top 10 causes of death. https://www.who.int/en/news-room/fact-sheets/detail/the-top-10-causes-of-death

Resende, A., Cláudia, A., Faria, M. De, De, A. C. M., Flávia, A., Necci, C., Wesley, D., Toledo, F., \& Rabelo, D. M. (2017). Atenção Farmacêutica No Monitoramento De Dislipidemias Secundárias a Partir De Diabetes, Obesidade E Alcoolismo. 2(1), 1-13.

Rodrigues, P. F., Melo, M., Assis, M., \& Oliveira, A. (2017). Condições socioeconômicas e prática de atividades físicas em adultos e idosos: uma revisão sistemática. Revista Brasileira de Atividade Física \& Saúde, 22(3), 217-232. https://doi.org/10.12820/rbafs.v.22n3p217-232

Souza, M. B. (2018). a Influência Da Mídia E Marketing Nos Hábitos Alimentares Infantis E O Comportamento Da Família. Revista Uniabeu, 11(29), 14-29.

World Health Organization. (2017). Cardiovascular diseases (CVDs). https://www.who.int/es/news-room/fact-sheets/detail/cardiovascular-diseases-(cvds) 